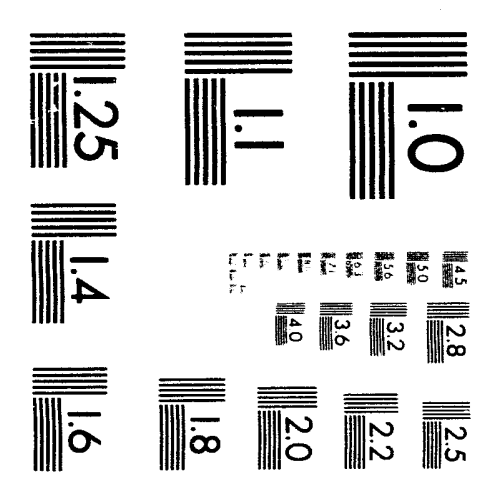



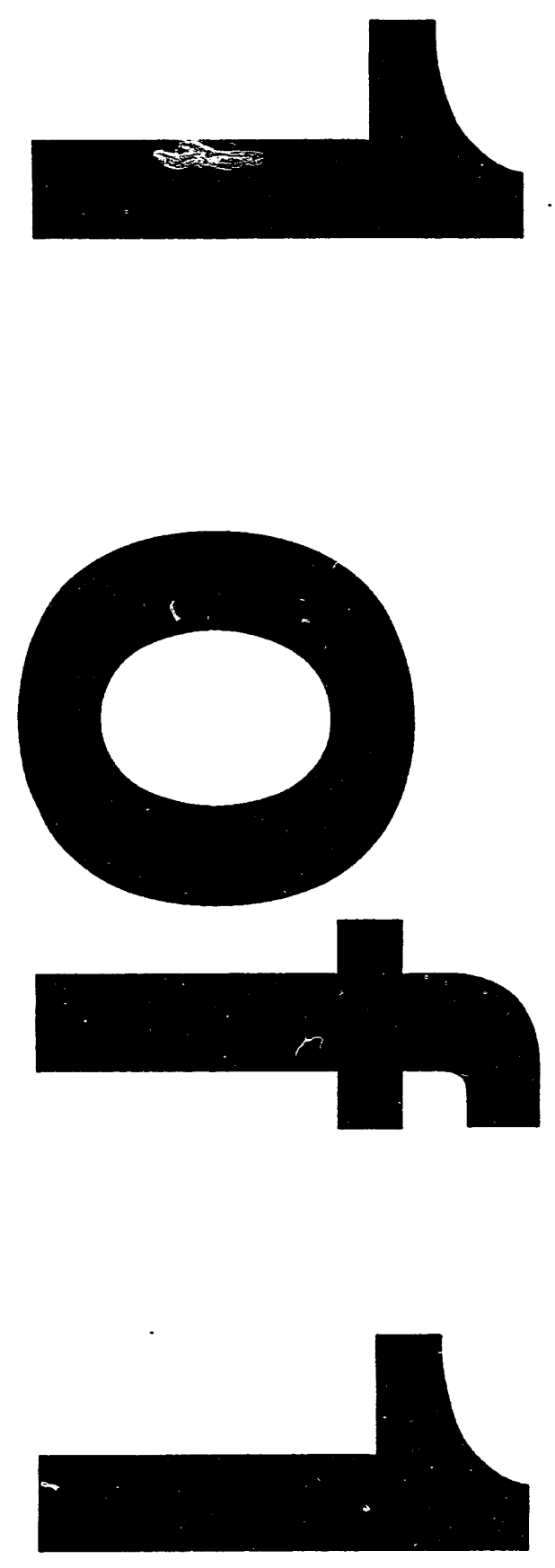


\section{$\operatorname{Cox} 6.9310208--1$}

\section{EXPERIMENTAL INVESTIGATIONS OF SENSOR-BASED SURFACE FOLLOWING TASKS BY A MOBILE MANIPULATOR*}

David B. Reister, Michael A. Unseren, James E. Baker, and François G. Pin Center for Engineering Systems Advanced Research

Oak Ridge National Laboratory P.O. Box 2008

Oak Ridge, TN 37831-6364
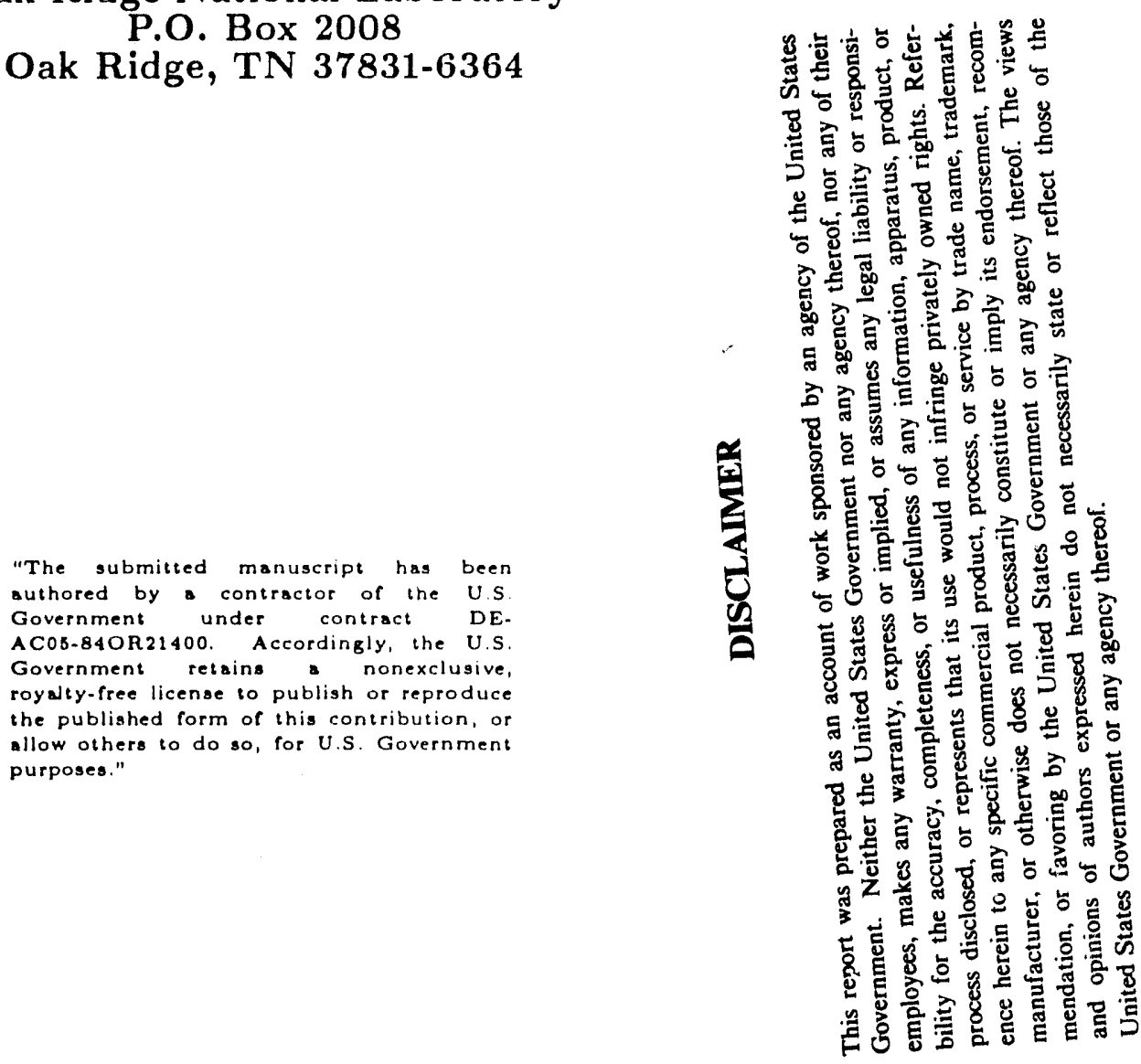

Submitted to: International Symposium on Experimental Robotics, October 27-29, 1993, Kyoto, Japan

* Research sponsored by the Engineering Research Program, Office of Basic Energy Sciences, of the U.S. Department of Energy, under contract DE-AC05-84OR21400 with Martin Marietta Energy Systems, Inc.

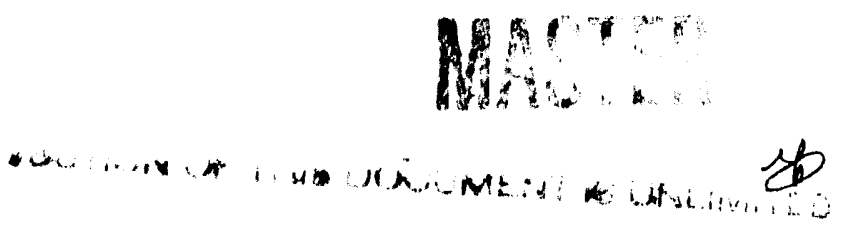




\title{
Experimental Investigations of Sensor-Based Surface Following Tasks by a Mobile Manipulator
}

\author{
David B. Reister, Michael A. Unseren, James E. Baker, and François G. Pin \\ Center for Engineering Systems Advanced Research \\ Oak Ridge National Laboratory \\ P.O. Box 2008 \\ Oak Ridge, TN 37831-6364
}

\begin{abstract}
This paper discusses experimental investigations of the feasibility and requirements of simultaneous external-sensor-based-control of the wheeled platform and the manipulator of a mobile robot. The experiments involve 3-D arbitrary surface following by the manipulator while the platform moves along a predefined trajectory.

A variety of concave and convex surfaces were used in the experiments, during which target and measured values of the platform and arm positions and orientations, together with the surface absolute location and normal estimates, were logged at $10 \mathrm{~Hz}$. For all experiments, the data logs showed significant noise, at high frequency, in the calculated surface normal values despite smooth tracking of their target values by the arm and the platform, with typical closed loop delays between target and achieved values of the order of $100 \mathrm{msec}$. This high-frequency noise in the calculated values is conjectured to result mainly from the arm's transmission cables compliance and backlash in the spherical wrist gears. On the other hand, the end-effector distance to the surface showed some low frequency errors of the order of $\pm 20 \%$. The two major sources of these low frequency errors appeared to reside respectively in the low values of the velocity bound and gain parameters utilized to filter the high frequency noise in the calculated normal values prior to using them as input to the arm control, and in the rolling contact of the platform's rubber-coated wheels on the ground where significant errors in the platform's positions and orientations can accumulate.
\end{abstract}

\section{Introduction}

Mobile manipulators, i.e., manipulators mounted on mobile platforms, are attracting significant interest in the industrial, military, and public service communities because of the significant increase in task capabilities and efficiency which results from their large-scale mobility combined with manipulation abilities. When the platform and manipulator move simultaneously, the motion planning ard control of the two subsystems cannot be fully decoupled; in particular the position estimations for trajectory tracking derived at loop rate from internal joint sensors need to be carefully integrated: while instantaneous position estimates are direct functions of the joint sensor data for the manipulator subsystem, they are functionals of the wheel rotation and steering sensor data for the platform, typically requiring time integration of these values over the entire trajectory history due to the non-holonomic nature of the wheeled system. As a result, motion accuracies of human-size platforms are generally two or three orders of magnitude worse than the accuracies of common manipulators. This paper discusses experiments performed with the HERMIES-III autonomous mobile manipulator,

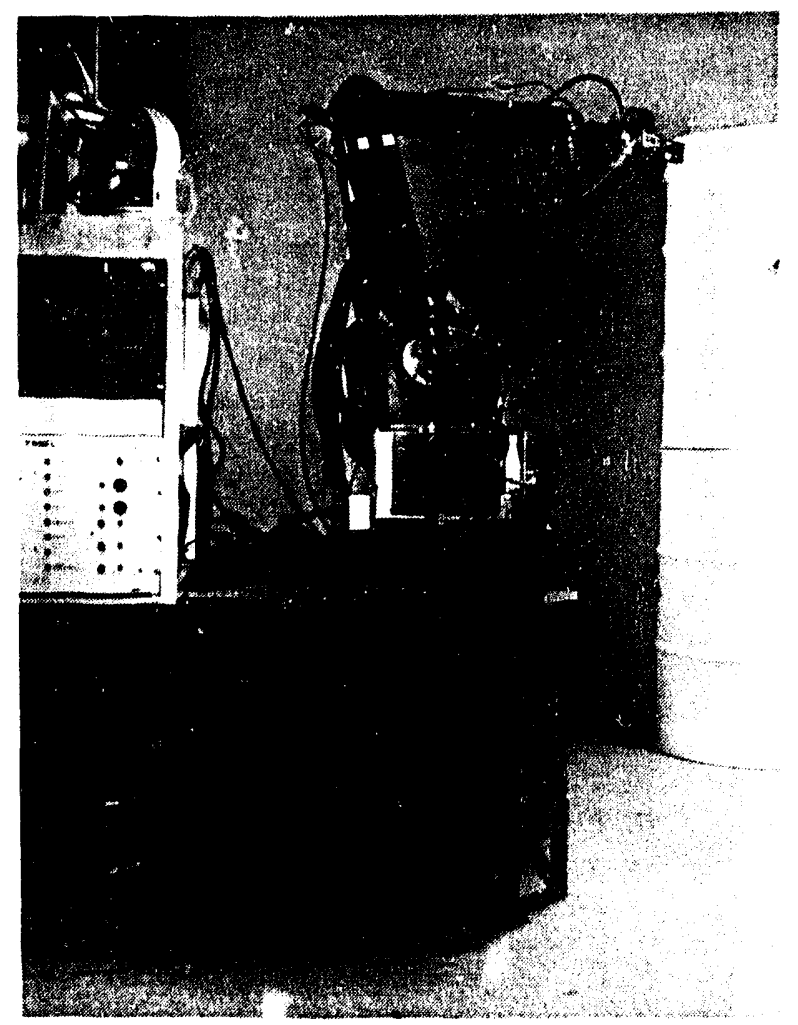

Fig. 1. The HERMIES-III test-bed robot, incorporating an omnidirectional platform and the seven degree-of-freedom CESARm manipulator, photographed during one of the surface following experiments. The single beam range finding sensor is held in the CFSARm's gripper. 
investigating the feasibility and requirements of simultaneous external-sensor-driven platform and redundant manipulator motions, and the interaction of the two subsystems' internal sensor-based position estimation. The experiments involved precise following of arbitrary 3-D surfaces by the end-effector of the redundant manipulator while the platform performs trajectory tracking along a predefined path.

\section{Experimental Configuration}

HERMIES-III [1],[2] is a human-size mobile manipulator test-bed (see Fig. 1) incorporating the seven degree-of-freedom (d.o.f.) CESARm research manipulator on a four d.o.f. platform (with two independently-driven and steerable wheels). The software and hardware configuration of IIERMIES-III utilizes the HELIX communication protocol [3], allowing for a fully distributed and message passing modular control system on several connected VMEbus racks. The platform's control drivers and sensor-feedback odometry modules operate at $20 \mathrm{~Hz}$. The redundancy resolution module for the CESARm includes $3-D$ position and 3-D orientation control and utilizes a minimum Euclidean norm-based algorithm running at $50 \mathrm{~Hz}$ on a 68020 processor [4]. The forward kinematics calculations run at $150 \mathrm{~Hz}$ on another 68020 processor.

For the experiments, a very accurate $(.02 \mathrm{~mm}$ precision), single point LED triangulation-type range finder [5],[6] was held in the gripper of the CESARm (see Fig. 1). The platform was assigned to perform a specified trajectory on the floor, while the CESARm end-effector's task was to follow an a priori unknown surface, maintaining both constant distance and constant orientation from the surface using the range finder data. Because the LED range sensor is unidirectional and provides data from a single beam, estimation of the surface normal (necessary to maintain constant orientation) required an estimate of the absolute displacement of the measurement point on the surface expressed in the reference (or world) coordinate system. This estimation therefore required propagation of position and orientation estimates through the entire platform-arm-gripper-sensor-beam chain.

\subsection{Surface Calculation}

The distance sensor is located on the end-effector of the CESARm that is mounted on the mobile platform of HERMIES-III. The goal is to measure the curvature of an arbitrary surface and keep the sensor at a fixed distance from the surface and normal to the surface. To measure the curvature of the surface, we must measure points on the surface in the world coordinate system. The system has three coordinate systems: world, platform, and arm (see Fig. 2). In this subsection we show how the measured data is used to calculate a point on the surface in each of the coordinate systems.

Our objective is to calculate a point on the surface of an object in the world coordinate system $\left(x_{s}, y_{s}\right)$. In the experiments dealt with here, we assume that the shape of the object does not depend on the $z$ coordinate (e.g., that the unknown object is a cylinder). Thus, all of our calculations will be in $2 \mathrm{D}$ geometry. The measured data are the distance to the surface (D), the configuration of the last link of the manipulator measured in the arm coordinate system $\left(x_{b}, y_{b}, \theta_{b}\right)$, and the location of the platform in the world coordinate system $\left(x_{p}, y_{p}, \phi_{p}\right)$. The arm is controlled in $3 \mathrm{D}$ space $(x, y, z$, roll, pitch, yaw) and $\theta_{b}$ is the measured yaw angle.

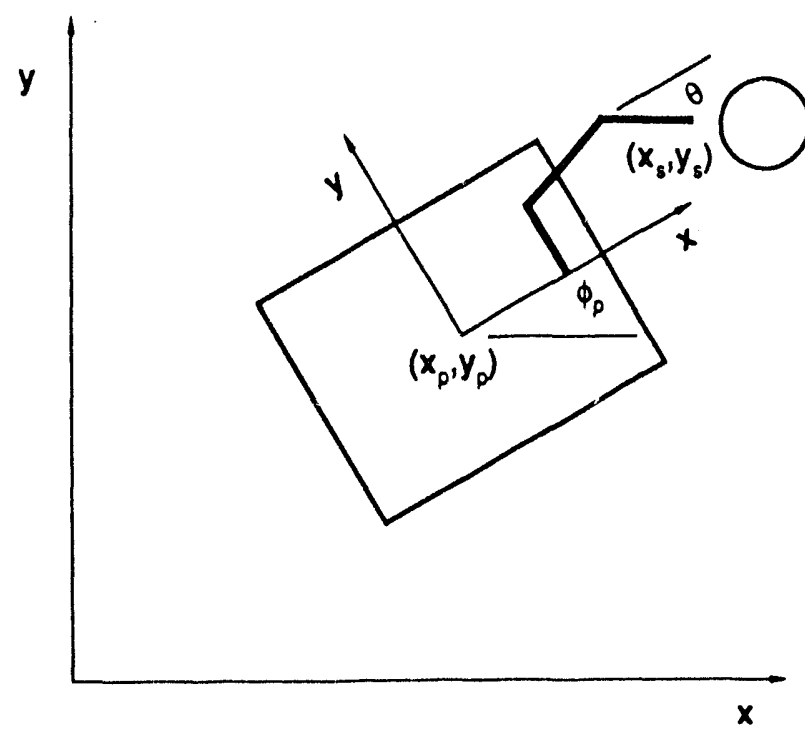

Fig.2. Schematic of the experimental configuration.

The base of the arm is attached to the platform. Thus, the transformation between the arm coordinates and the platform coordinates does not vary. Unfortunately, the transformation requires both a translation and a rotation of 180 degrees. If $\left(x_{h}, y_{h}, \theta_{h}\right)$ is the configuration of the last link of the manipulator measured in the platform coordinate system:

$$
\begin{gathered}
x_{h}=-x_{b}+\mathrm{B} \\
y_{h}=-y_{b} \\
\theta_{h}=\theta_{b}-\pi / 2
\end{gathered}
$$

where $\mathrm{B}$ is the $x$ coordinate of the arm base in the platform coordinate system ( $B=0.574$ meters). In the home position for the arm, $\left(x_{b}, y_{b}, \theta_{b}\right)=(-0.880$, $-0.356, \pi / 2)$ and $\left(x_{h}, y_{h}, \theta_{h}\right)=(1.454,0.365,0.0)$.

To reach the surface of the object in the platform coordinate system $\left(x_{d}, y_{d}\right)$, we travel in the $\theta_{h}$ direction by the sum of the length of the distance sensor ( $L=0.062$ meters) and the measured distance (D):

$$
x_{d}=x_{h}+(D+L) \cos \theta_{h}
$$




$$
y_{d}=y_{h}+(D+L) \sin \theta_{h}
$$

To calculate a point $\left(x_{s}, y_{s}\right)$ on the surface of the object in the world coordinate system, we transform the coordinates from the platform reference frame to the world reference frame as follows:

$$
\begin{aligned}
& x_{s}=x_{p}+s_{d} \cos \phi_{p}-y_{d} \sin \phi_{p} \\
& y_{s}=y_{p}+x_{d} \sin \phi_{p}+y_{d} \cos \phi_{p}
\end{aligned}
$$

Note that we have used all of the measured data $\left[D,\left(x_{b}, y_{b}, \theta_{p}\right)\right]$ to calculate the point location on the surface $\left(x_{s}, y_{s}\right)$.

\subsection{Surface Normal Calculation}

The location of the points on the surface are calculated at $100 \mathrm{~Hz}$. At $10 \mathrm{~Hz}$, we would like to calculate the surface normal and the arm goal. To calculate the surface normal, we fit a polynomial to the surface points and calculate the slope of the curve. There are tradeoffs in choosing the order of the polynomial and the number of data points to use to estimate the parameters. A higher order polynomial (cubic or quadratic) has more parameters and requires more data points to estimate the parameters. Furthermore, a high order polynomial might not provide a good fit to a surface with discontinuous surface normals (e.g., a box). Our goal is to obtain the best estimate of the surface normal in a small neighborhood of the currently measured point on the surface. Thus, we would like to fit a curve with a small number of parameters using a small number of data points. We decided to fit a line using $M$ points (currently, $M=10$ ), where $M$ is a user-defined parameter that can be adjusted to improve performance.

Given $M$ points on the surface, we determine the maximum and minimum values for each of their coordinates, $x$ and $y$. If the spread in $x$ is greater than the spread in $y$, we assume that $y=f(x)$. Otherwise, we assume that $x=f(y)$. The maximum speed for the platform is 0.45 meters/second. Thus, the maximum distance traveled in 0.1 seconds is 0.045 meters. If the maximum spread is less than 0.003 meters, we do not calculate the surface normal. Otherwise, we use least squares to fit a line to the data points.

Let $\psi$ be the normal to the line and let $\theta_{s}$ be the surface normal in the platform coordinate system, then:

$$
\theta_{s}=\psi-\phi_{p}
$$

Typical experimental values for the surface normal $\left(\theta_{s}\right)$ are displayed in Fig. 3.

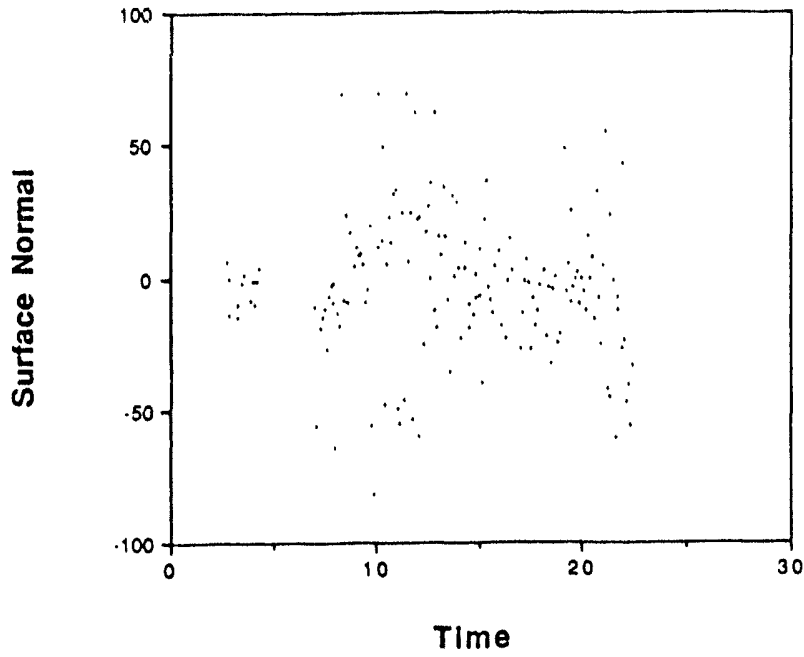

Fig. 3. Actual time log of the surface normal calculation (in degree) .

It is clear that the values displayed in Fig. 3 are much too variable or noisy to be fed as input target to the manipulator. When the input to the arm has high frequency noise, the arm will oscillate. To provide a smooth input to the arm, we filtered the calculated values of the surface normal $\left(\theta_{s}\right)$. Let $\theta_{g}$ be a running average of the calculated values for the surface normal:

$$
\theta_{g}(t+1)=(1-\mu) \theta_{g}(t)+\mu \theta_{s}(t)
$$

When the parameter $\mu$ is 1.0 , the average value is equal to the input $\left(\theta_{g}=\theta_{s}\right)$. When the parameter $\mu$ is 0.0 , the average is a constant that does not depend on the input. The current value for the parameter is $\mu=0.1$.

Let $\theta_{c}$ be the target value for the orientation of the arm. We let the target track the average value of the surface normal. Let $\epsilon$ be the difference between the target and the average value: $\epsilon=\theta_{g}-\theta_{c}$. We limited the allowable rate of change $\epsilon$ to a given value $\delta$ (currently, $\delta=0.01$ ). Thus, if $\epsilon>\delta$, then $\epsilon=\delta$ and if $\epsilon<-\delta$, then $\epsilon=-\delta$. Finally:

$$
\theta_{c}(t+1)=\theta_{c}(t)+\epsilon
$$

Thus, the three parameters $(M, \mu$, and $\delta)$ can be used to smooth the time varying input to the arm.

\subsection{Arm Goal Position Calculation}

The calculation of a goal for the arm is illustrated in Fig. 4. The current orientation of the $\operatorname{arm}$ is $\theta$. In the current position, the schematic follows the arm from the wrist $(w)$, to the hand $(h)$, and past the surface detector $(d)$ to the surface $(s)$. The figure also displays the goal configuration of the arm (at orientation $\theta_{c}$ ). Given the orientation, the goal for the hand $\left(x_{c}, y_{c}\right)$ is calculated as:

$$
x_{c}=x_{d}-\left(D_{g}+L\right) \cos \theta_{c}
$$




$$
y_{c}=y_{d}-\left(D_{g}+L\right) \sin \theta_{c}
$$

where the desired value for the distance is $D_{g}=0.102$ meters in the experiments described here.

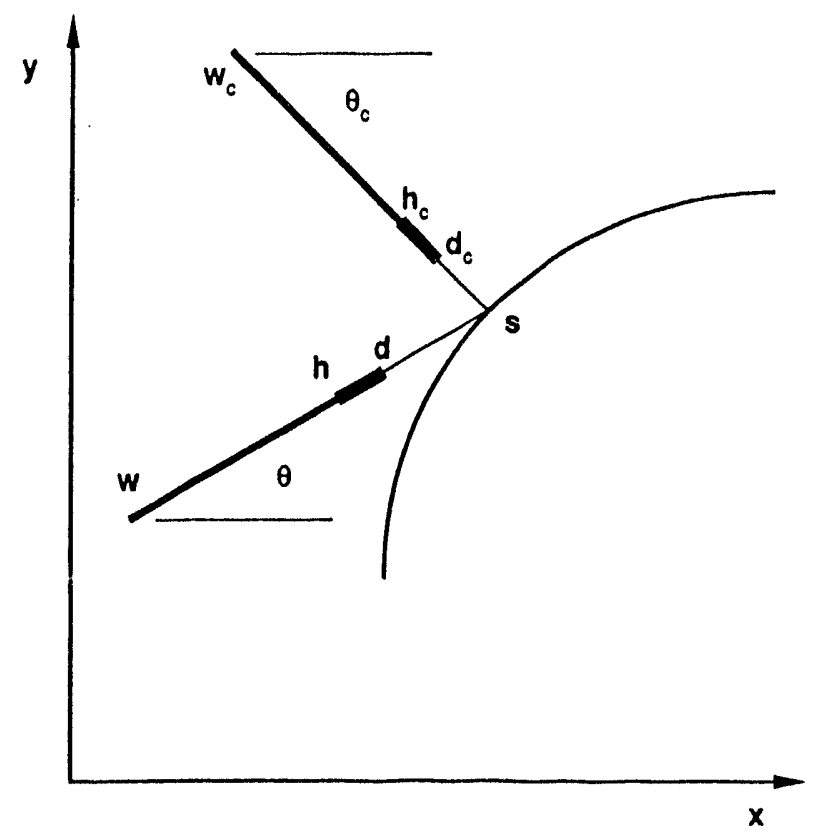

Fig. 4. Schematic of the arm-wrist-sensor chain for the calculation of a goal position and orientation $\left(W_{c}\right)$ for the end-effector from measurements in previous positions.

\section{Experimental Results and Discussion}

The data displayed in this section (and in Fig. 3) were collected during an experiment in which the platform moved in an arc about a barrel and returned to its initial location. Figure 5 displays the distance measurements from the range finder during the experiment. The goal is to maintain a distance of 0.102 meters. Clearly, the measured values show that the end-effector can be more than two centimeters from the goal. The low frequency of the curve would seem to indicate that an error accumulation takes place over time in the calculational system resulting in a gradual drift of some of the components from their target values.

The manipulator arm was first investigated as the possible source of the error. As seen below, the manipulator system was consistently following the target values for both position and orientation as calculated from Eqs. (1) to (12). As an example of this, Fig. 6 displays a time log of the target $\left(\theta_{c}+\pi / 2\right)$ and the measured values $\left(\theta_{b}\right)$ for the manipulator yaw angle. The targets move smoothly and the measured values follow the targets accurately, with an expected lag in execution of the order of one to two cycles of the $10 \mathrm{~Hz}$ calculational scheme. This time delay between the targets (set points) and the execution, however, could not explain either the low frequency divergences exhibited in Fig. 6 or the very large scattering displayed in Fig. 3. Our conjecture here is that some of the low frequency errors already are present in the target themselves, due mainly to the values of the smoothing and bounding parameters $\mu$ and $\delta$ used in Eqs. (9) and (10).

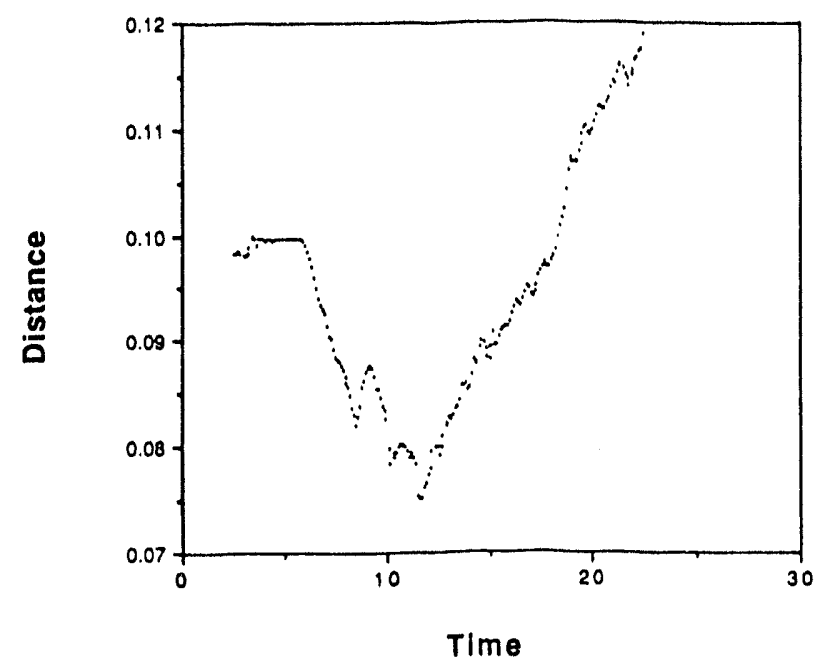

Fig. 5. Time log of the distance between the end-effector and the surface measured by the range finder sensor.

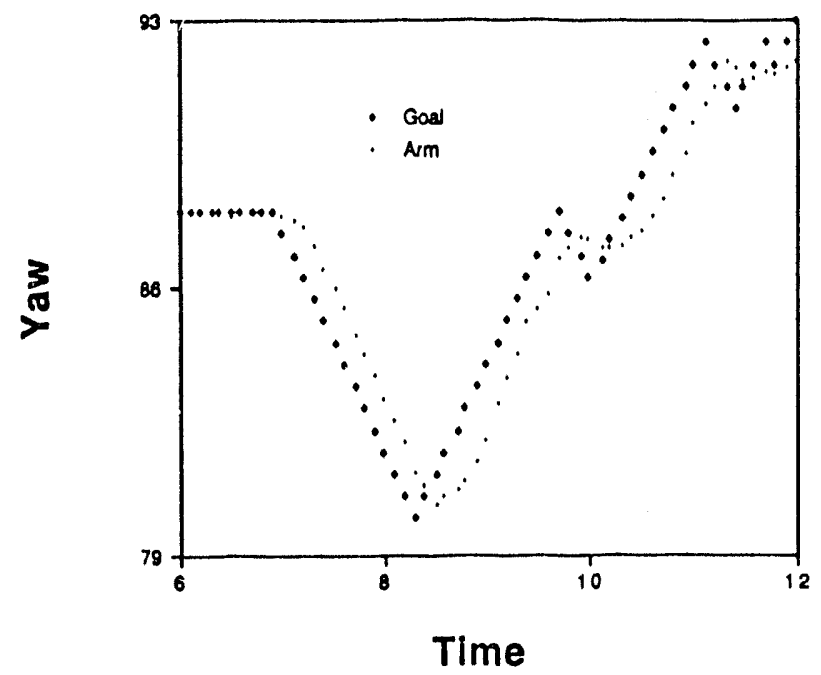

Fig. 6. Sample time log of the target and measured values of the end-effector yaw angle.

The data from the platform's encoders and odometry calculations showed results very similar to those obtained for the arm: smooth, although slightly delayed, tracking of the target values by the measured values. Since the platform appeared to respond accurately to its local target, the low frequency error is conjectured to result from an error accumulation not directly detectable by the platform sensors. 
Figure 7 is a plot of the points $\left(x_{s}, y_{s}\right)$ on the surface of the barrel calculated in the world reference frame from integration of the measured data over the entire platform-arm-sensor chain. There is significant noise in the data and both the low and high frequency errors can be observed. The very large scattering in the surface normal results which were observed in Fig. 3 correlate with the high frequency variations observed on Fig. 7. A possible solution to this high frequency problem could reside in a better smoothing of the measured range data and surface estimation using the three parameters $(M, \mu$, and $\delta)$ described in the previous section, in such a way however that the effect of the bounding parameter $\delta$ on the low frequency error of the system is minimized. Some of our future work includes a set of systematic experiments to determine optimum values for these parameters.

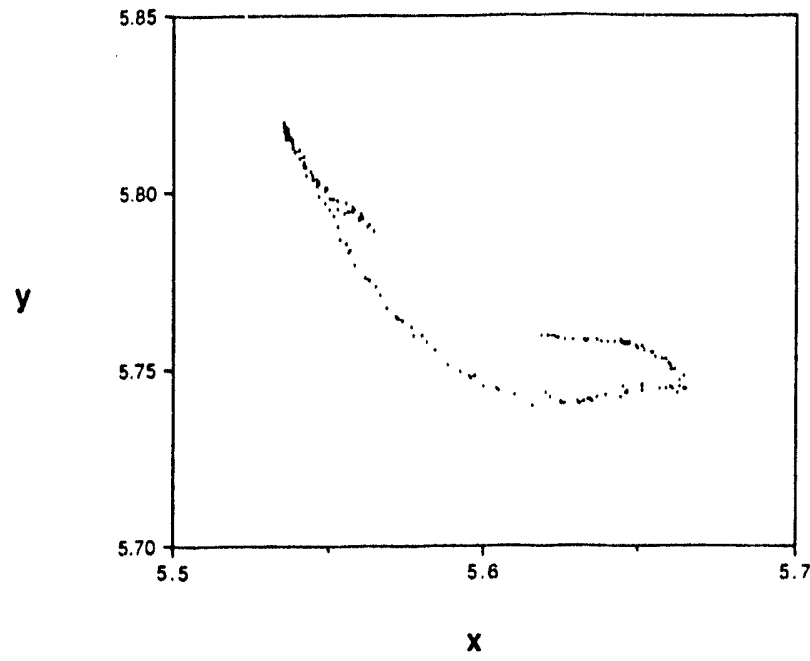

Fig. 7. Display of the calculated absolute location of the followed surface.

A large part of the low frequency errors, however, cannot be corrected through any type of filtering. Although the reckoner for the platform can produce slight integration errors, the major sources of the large observed errors in Fig. 7 are thought to reside in the rolling contact of the platform's wheel on the floor. When moving along a circle, with the two driving wheels steered at different angles, the effective point of contact of the wide rubber-coated wheels varies slightly under the wheels with slight irregularities on the floor. This generates errors in the wheel velocity targets that are calculated to fulfill the rigid body constraint (which exists between the wheels in this configuration). From these errors, slight slippage of the wheels on the floor results, progressively accumulating to large errors, undetectable with the odometry sensors.

In a first step toward remedying this problem, a composite control architecture which accommodates for violations of the interwheel rigid body kinematic constraints in addition to controlling the position of the platform wheel system has recently been developed [7] and has shown dramatic improvements on the platform's control accuracy when used on HERMIES-III. The architecture, however, is based on a kinematic model of the platform and does not account for the inertia required to induce motion. This could be another source of error, and is the focus of some of our future investigations. Another improvement in the system's control involves decoupling the sensor-based control of the end-effector's distance-to-the-surfacr: and the calculations of the surface normal estimates, performing the former entirely in the manipulator frame of reference, while keeping the latter in the absolute reference frame. This effectively decouples the filtering process of the high frequency noise in the calculated values, such as the surface normal, from some of the sensor-based feedback control, therefore allowing for greater flexibility in the range of smoothing and gain parameters.

\section{Acknowledgment}

This research was performed under sponsorship of the Engineering Research Program, Office of Basic Energy Sciences, of the U.S. Demartment of Energy, under contract DE-AC05-84OR21400 with Martin Marietta Energy Systems, Inc.

\section{References}

[1] F. G. Pin et al., "Autonomous Mobile Robot Research Using the HERMIES-III Robot," Proceedings of the IEEE RSJ International Workshop on Intelligent Robots and Systems, IROS '89, September 4-6, 1989, Tsukuba, Japan, 251-256.

[2] D. B. Reister, "A New Wheel Control System for the Omnidirectional HERMIES-III Robot," Robotica 10, 351-360 (1992).

[3] J. P. Jones et al., "Hetero Helix: Synchronous and Asynchronous Control Systems in Heterogeneous Distributed Networks," Robotics and Autonomous Systems 10(2-3), 85-99 (1992).

[4] M. A. Unseren, "Input Relegation Control for Gross Motion of a Kinematically Redundant Manipulator," Oak Ridge National Laboratory Technical Report No. ORNL/TM-12165, October 1992.

[5] J. E. Baker, "Empirical Characterization of a High Intensity Proximity Sensor," Sensors 10(7), 29-32 (1993).

[6] J. E. Baker, "Terrain Following and Mapping of Arbitrary Surfaces Using High Precision Proximity Data," Optical Engineering 32(5), 1117-1124 (1993).

[7] D. B. Reister and M. A. Unseren, "Position and Constraint Force Control of a Vehicle with Two or More Steerable Drive Wheels," accepted for publication in IEEE Transactions on Robotics and Automation. 

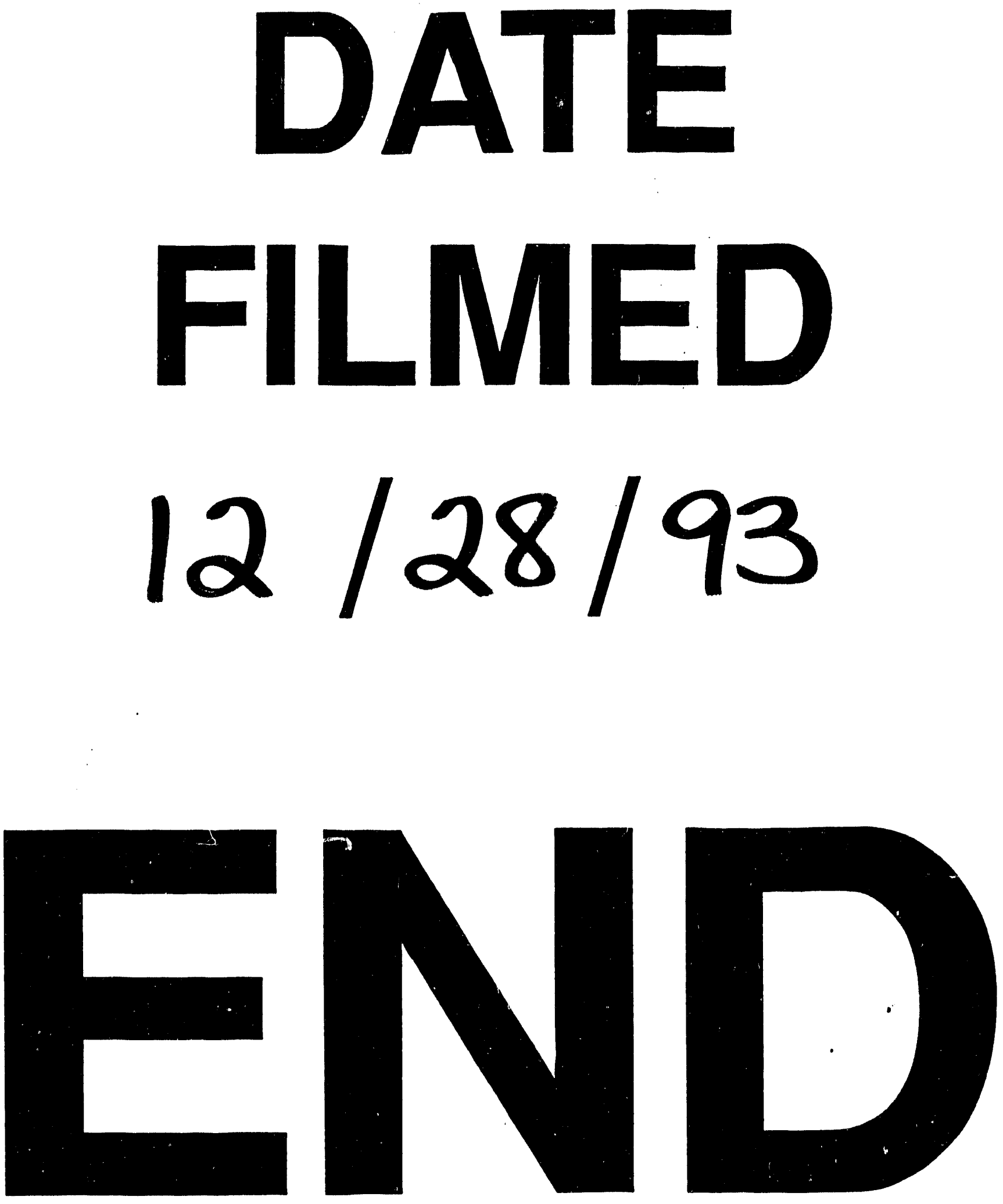
